\title{
Mechanisms of Enhanced Antigen Delivery to Murine Dendritic Cells by the Cationic Liposomes
}

\author{
Saeko Takahashi, Rui Tada*, Yoichi Negishi, Yukihiko Aramaki \\ Department of Drug Delivery and Molecular Biopharmaceutics, School of Pharmacy, Tokyo University of Pharmacy and Life \\ Sciences, Tokyo, Japan \\ Email: *rui.tada@gmail.com
}

How to cite this paper: Takahashi, S., Tada, R., Negishi, Y. and Aramaki, Y. (2017) Mechanisms of Enhanced Antigen Delivery to Murine Dendritic Cells by the Cationic Liposomes. Open Journal of Immunology, 7, 85-101.

https://doi.org/10.4236/oji.2017.74007

Received: November 22, 2017

Accepted: December 15, 2017

Published: December 19, 2017

Copyright $\odot 2017$ by authors and Scientific Research Publishing Inc. This work is licensed under the Creative Commons Attribution International License (CC BY 4.0).

http://creativecommons.org/licenses/by/4.0/

\section{(c) (i) Open Access}

\begin{abstract}
There is an increased demand for vaccines to prevent and/or treat illness and mortality caused by the infectious diseases. We have recently established that liposomes composed of cationic lipids act as adjuvant for nasal vaccine formulation. However, the molecular mechanism(s) behind the adjuvant effect remain unrevealed. To this end, we have studied the enhancement of antigen uptake by murine dendritic cell line, DC2.4 cells, by the cationic liposomes and the specific pathways involved in the process. We have observed that the uptake of ovalbumin (OVA) into DC2.4 cells is greatly increased when co-cultured with the cationic liposomes composed of 1,2-dioleoyl-3-trimethylammonium-propane (DOTAP) and $3 \beta$-[N-(N',N'-dimethylaminoethane)-carbamoyl] (DC-chol). However, this enhancement was blocked by pretreatment of DC2.4 cells with chlorpromazine and methyl- $\beta$-cyclodextrin, indicating the involvement of clathrin- and caveolin-independent lipid raft-dependent endocytic pathways in the process. Our results implied, at least in part, that enhanced uptake of antigens induced by the cationic liposomes could be a possible mechanism for the induction of immune responses. Although further studies are needed to understand the precise mechanisms behind the adjuvant effects of DOTAP/DC-chol liposome, this approach is quite useful for the development of vaccine system to combat various diseases.
\end{abstract}

\section{Keywords}

Antigen Delivery, Cationic Liposome, Dendritic Cells, Nanoparticle

\section{Introduction}

The demand for vaccines is greatly increased, as vaccination seems to be the only 
approach in preventing and/or treating illness and mortality associated with infectious diseases [1] [2]. Despite that the traditional vaccine systems, such as live-attenuated and inactivated vaccines, are quite effective, their clinical usage for deadly pathogens have still been challenging because of the safety concerns and risks for infections [3] [4] [5]. Recently, there has been an emphasis on new generation subunit vaccines due to its safety features compared to both live-attenuated and inactivated vaccines [6] [7]. However, for these subunit vaccines, an adjuvant component, which potentiates host immunity, is crucial for the effective induction of antigen-specific immune responses as the antigenic property of proteins are inherently poor when administered to the host [8]. While numerous adjuvants have been explored experimentally, their translation to clinical usage in humans is extremely slow. Thus a clinical need for the development of novel adjuvants arises. Adjuvants generally exhibit their immune-enhancing activities to the antigens through the following mechanisms: 1) the depot effect by which releases antigen from adjuvant carriers at the site of injection gradually, 2) activation of host innate immune responses and 3) increased antigen uptake by antigen-presenting cells (APCs) and in turn its presentation to T cells [9].

Dendritic cells (DCs) are one of the most favored targets for vaccine delivery because of their primary role in the initiation of acquired immune responses via antigen internalization followed by antigen processing and presentation to lymphocytes, especially to $\mathrm{T}$ cells via the major histocompatibility complex (MHC) molecules [10] [11]. Nanoparticles such as liposomes and gold nanoparticles are now considered to be not only efficient drug carriers but also potent adjuvants useful for vaccine formulations. For example, a nanogel composed of cholesteryl-group-bearing pullulan acts as an adjuvant by promoting antigen delivery to dendritic cells [12] [13]. Similarly, a well-known carrier for nucleic acid delivery, polyethyleneimine (PEI), is reported to be a potent adjuvant as the release of host double-stranded DNA (dsDNA) into the extracellular milieu triggers Ifr3-dependent innate immune signaling [14].

Cationic liposome is recognized as a powerful tool for vaccine development as well as for nonviral gene delivery system [15] [16] [17] [18]. We recently demonstrated that the cationic liposomes composed of

1,2-Dioleoyl-3-trimethylammonium-propane (DOTAP) and

$3 \beta$-[N-(N',N'-dimethylaminoethane)-carbamoyl] (DC-chol), termed as

DOTAP/DC-chol liposomes, are served as a potent adjuvant. The detailed molecular mechanism(s) behind the adjuvant effects of the cationic liposomes are not completely elucidated. We have previously reported that cationic liposome promotes antigen uptake into the DCs located at nasal-associated lymphoid tissues (NALTs) in vivo [19] [20]. Although, how the cationic liposome increases antigen uptake by DCs remains unclear till date. In general, the difference in the uptake mechanism(s) of particulate and soluble forms of antigenic proteins by DCs might be the reason behind this increase in uptake efficiency of antigens 
tagged to a delivery system [21] [22]. Understanding this process might be crucial for optimization of liposomal design, including its features like particle size and lipid composition, for inducing maximum immune responses.

The goal of this study is to understand the molecular mechanisms involved in the increase in antigen uptake into DCs by the cationic liposomes. Here, we investigated the role of various endocytic pathways on enhanced ovalbumin (OVA) uptake into murine dendritic cell lines, DC2.4, mediated by the cationic liposomes by perturbing various endocytic pathways using specific chemical inhibitors.

\section{Materials and Methods}

\subsection{Cells and Reagents}

DC2.4 cells were kindly gifted to us by Dr. Jun Kunisawa at the National Institutes of Biomedical Innovation, Health and Nutrition (NIBIOHN, Osaka, Japan). DOTAP and DC-chol were purchased from Avanti Polar Lipids (Alabaster, AL, USA). 1,1'-Dioctadecyl-3,3,3',3'-tetramethylindocarbocyanine perchlorate (DiI) was purchased from Thermo Fisher Scientific (Waltham, MA, USA). Fluorescein-4-isothiocyanate (FITC) was obtained from Dojindo (Kumamoto, Japan). Low endotoxin (less than $1 \mathrm{EU} / \mathrm{mg}$ guaranteed) egg white OVA, heparin sodium, genistein, and sodium azide were acquired by Wako Pure Chemical Industries (Osaka, Japan). 3-(4,5-Dimethyl-2-thiazolyl)-2,5-diphenyltetrazolium Bromide thiazolyl blue (MTT) was obtained from NacalaiTesque (Kyoto, Japan). 4',6-diamidino-2-phenylindole (DAPI), chlorpromazine hydrochloride, cytochalasin D, and methyl-cyclodextrin were purchased from Merck (Darmstadt, Germany). Amiloride hydrochloride was purchased from Cayman Chemical Company (Ann Arbor, MI, USA).

\subsection{Preparation of Cationic Liposomes}

Liposomes were prepared as described earlier [19]. Briefly, $10 \mu \mathrm{mol}$ of total lipids (DOTAP:DC-chol at 1:1 mol ratios) were evaporated to dryness in a glass tube and desiccated for at least $1 \mathrm{~h}$ in vacuo. The resultant lipid films were hydrated by the addition of $250 \mu$ l of phosphate-buffered saline (PBS; Wako Pure Chemical Industries, Tokyo Japan)) and then vortexed for $5 \mathrm{~min}$ at room temperature (RT). The prepared multilamellar vesicles were extruded 10 times by passing through a $100 \mu \mathrm{m}$-pore polycarbonate membrane (Advantec, Tokyo, Japan) and then sterilized by filtration through $0.45 \mu \mathrm{m}$ filter membranes (Iwaki, Tokyo, Japan). The particle size and $\zeta$-potential of the liposomes were measured by Nicomp 380 ZLS (Particle Sizing Systems; Port Richey, USA). The liposomes used in this study had a particle size of $149.3 \pm 5.5 \mathrm{~nm}$ with a $\zeta$-potential of $8.9 \pm$ $2.0 \mathrm{mV}$. The fluorescent-labeled cationic liposomes (DiI liposomes) were prepared in the same way by combining $0.2 \%$ DiI with $10 \mu \mathrm{mol}$ of DOTAP:DC-chol 1:1 mixture. The DiI liposomes prepared here had a particle size of $143.2 \pm 5.0$ $\mathrm{nm}$ with a $\zeta$-potential of $9.8 \pm 2.0 \mathrm{mV}$. 


\subsection{Preparation of Fluorescein-4-Isothiocyanate (FITC)-Labeled OVA}

FITC-labeled OVA was prepared by the method described previously [23]. Briefly, $5 \mathrm{mg}$ of FITC in $0.4 \mathrm{~mL}$ of DMSO was added drop-wise to $4 \mathrm{~mL}$ of OVA $(50 \mathrm{mg} / \mathrm{mL})$ in $0.1 \mathrm{M}$ sodium carbonate buffer ( $\mathrm{pH} 9.0)$ with gentle agitation, in dark. After $8 \mathrm{~h}$ incubation at $4^{\circ} \mathrm{C}$, the reaction was quenched by the addition of ammonium chloride to a final concentration of $50 \mathrm{mM}$ and incubated further for $2 \mathrm{~h}$ to obtain the crude FITC-OVA. The conjugate was then purified by gel filtration chromatography using Toyopearl HW-55F (Tosoh Corporation, Tokyo, Japan) resin. The protein concentration of purified FITC-OVA preparation was determined by the BCA Protein Assay Kit (Wako Pure Chemical Industries).

\subsection{In Vitro Cytotoxic Assay}

The cytotoxic effects of the liposomes on DC2.4 cells were examined by an MTT assay. Briefly, DC2.4 cells were maintained in RPMI 1640 medium (Wako Pure Chemical Industries) supplemented with 10\% heat-inactivated fetal bovine serum (FBS; Biowest, Nuaillé, France), $100 \mu \mathrm{g} / \mathrm{mL}$ of streptomycin sulfate salt (Merck), and $100 \mathrm{U} / \mathrm{mL}$ of penicillin $\mathrm{G}$ potassium salt (Merck). The cells were cultured in a 96-well flat-bottom plate (Thermo Fisher Scientific) at a cell density of $5 \times 10^{4}$ cells/well in $0.1 \mathrm{~mL}$ culture media containing the liposomes $(0-400$ $\mathrm{nmol} / \mathrm{mL}$ ) for $48 \mathrm{~h}$ at $37^{\circ} \mathrm{C}$ in a humidified atmosphere containing $5 \% \mathrm{CO}_{2}$ and 95\% air. After incubation, the cells were centrifuged and washed twice with fresh RPMI 1640 medium and treated with $0.5 \mathrm{mg} / \mathrm{mL}$ of MTT (dissolved in PBS and filter sterilized by a $0.22 \mu \mathrm{m}$ PES membrane) for $2 \mathrm{~h}$ at $37^{\circ} \mathrm{C}$. The intracellular formazan crystals formed by the reduction of MTT by viable cells were dissolved in $0.01 \mathrm{M} \mathrm{HCl}$ solution containing $10 \%$ sodium dodecyl sulfate (SDS) to form a purple color. The optical density was measured at $570 \mathrm{~nm}$ using $650 \mathrm{~nm}$ as reference. The intensity of the color in each well is directly proportional to the number of viable cells. The cell viability in liposome treated DCs was calculated as the percentage (\%) with respect to the untreated control group.

\subsection{Flow Cytometry}

DC 2.4 cells were cultured in RPMI 1640 medium as described in section 2.5. The cells were plated on a 24 -well flat bottom plate at $5 \times 104$ cells/well density. After $24 \mathrm{~h}$, FITC-OVA $(50 \mu \mathrm{g} / \mathrm{mL})$, DiI-liposomes $(100 \mathrm{nmol} / \mathrm{mL})$, or FITCOVA $(50 \mu \mathrm{g} / \mathrm{mL})$ plusDiI-liposomes $(100 \mathrm{nmol} / \mathrm{mL})$ were added to the each well and incubated for $1 \mathrm{~h}$ at $37^{\circ} \mathrm{C}$ in the presence or absence of any of the following chemical inhibitors for endocytic pathways: genistein $(25 \mathrm{nM})$, sodium azide (10 $\mu \mathrm{M})$, chlorpromazine hydrochloride $(50 \mu \mathrm{M})$, cytochalasin $\mathrm{D}(2.5 \mu \mathrm{M})$, methyl- $\beta$-cyclodextrin $(31.25 \mu \mathrm{M})$, and Amiloride hydrochloride $(50 \mu \mathrm{M})$. The culture supernatant was removed and the cells were harvested by treatment with 1 $\mathrm{mM}$ ethylenediaminetetraacetic acid (EDTA) in PBS (Nacalai Tesque). The cells 
were carefully washed thrice with PBS containing heparin sodium $(100 \mu \mathrm{g} / \mathrm{mL})$ to remove residual DiI-liposomes, if any, from cell surface. The cells were analyzed using a FACSCantoII (BD Biosciences, Franklin Lakes, NJ, USA) to assess internalization of FITC-OVA and DiI-liposome. The mean fluorescence intensity (MFI) was quantified using Flowlogic software (Inivai Technologies, Mentone Victoria, Australia).

\subsection{Confocal Imaging}

DC2.4 cells, plated on a poly-L-lysine (PLL)-coated glass plate (Matsunami Glass, Osaka, Japan) at a cell density of $5 \times 10^{4}$ cells/well were used for confocal imaging. The cells were cultured in RPMI 1640 medium supplemented with 10\% heat-inactivated FBS, $100 \mu \mathrm{g} / \mathrm{mL}$ of streptomycin sulfate salt, and $100 \mathrm{U} / \mathrm{mL}$ of penicillin $\mathrm{G}$ potassium salt and incubated for $24 \mathrm{~h}$ at $37^{\circ} \mathrm{C}$ in a humidified atmosphere containing $5 \% \mathrm{CO}_{2}$ and $95 \%$ air. Then, FITC-OVA $(50 \mu \mathrm{g} / \mathrm{mL})$ alone, DiI-liposomes $(100 \mathrm{nmol} / \mathrm{mL})$ alone, or FITC-OVA $(50 \mu \mathrm{g} / \mathrm{mL})$ plusDiI-liposomes $(100 \mathrm{nmol} / \mathrm{mL})$ were added to the each well and incubated for $1 \mathrm{~h}$ at $37^{\circ} \mathrm{C}$ in the presence or absence of chemical inhibitors, chlorpromazine hydrochloride $(50 \mu \mathrm{M})$, methyl- $\beta$-cyclodextrin $(31.25 \mu \mathrm{M})$, or amiloride hydrochloride $(50 \mu \mathrm{M})$. Afterwards the cells were washed thrice with PBS containing heparin sodium $(100 \mu \mathrm{g} / \mathrm{mL})$ and fixed in $4 \%$ paraformaldehyde for $1 \mathrm{~h}$ at $4^{\circ} \mathrm{C}$. The fixed cells were washed extensively and were mounted in $90 \%$ glycerol in PBS containing $1 \mu \mathrm{g} / \mathrm{mL}$ of DAPI for nuclear staining. Confocal images were acquired using an FV1000-D confocal microscope (Olympus, Tokyo, Japan).

\subsection{Statistical Analysis}

Statistical differences in cytotoxicity assay and internalization assay were assessed using one-way ANOVA with Bonferroni's post-hoc test and unpaired $t$-test with Welch's correction, respectively.

\section{Results}

\subsection{Toxicity of the Cationic Liposomes on Murine Dendritic Cell Line, DC2.4}

It has been reported previously that the cationic liposomes generally show cytotoxicity against various types of cells [24] [25] [26] [27]. Hence, we have studied the cellular toxicity of cationic liposomes on DC2.4 cells in vitro to determine the optimal concentration of cationic liposomes to be used for further experiments. The viability of cells treated with various concentrations of cationic liposomes was assessed using an MTT assay. The cationic liposomes show a significant cellular toxicity on DC2.4 cells evidenced by a reduction in living cells $(\sim 50 \%)$ at concentrations above $200 \mathrm{nmol} / \mathrm{mL}$. Whereas the lower concentrations of cationic liposomes (below $100 \mathrm{nmol} / \mathrm{mL}$ ) did not show any cytotoxicity against DC2.4 cells in vitro (Figure 1). Therefore, we have used the cationic liposomes at $100 \mathrm{nmol} / \mathrm{mL}$ concentration for further experiments in this study. 


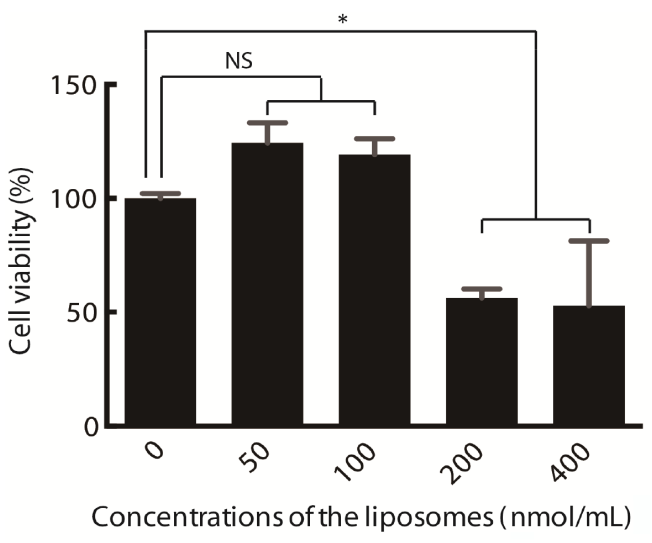

Figure 1. Cytotoxicity of the cationic liposomes on DC2.4 cells. DC2.4 cells were cultured for $48 \mathrm{~h}$ in the presence or absence of various concentrations of the cationic liposome. The cell viabilities were then assessed by the MTT assay. The cell viability (\%) was calculated on the basis of the ratio to the untreated group. The values are expressed as the mean \pm SD of triplicate cultures from three independent experiments. Significance was evaluated by a one-way ANOVA with Boferroni's post-hoc test: ${ }^{\star} p<0.05$, N.S.: not significant.

\subsection{Effect of the Cationic Liposomes on OVA Uptake by DC2.4 Cells}

We evaluated whether the cationic liposome promotes OVA uptake by DC2.4 cells in vitro. The cationic liposomes markedly increased the uptake of OVA by DC2.4 cells compared to an OVA alone-treated group as evidenced by an increase in MFI (39.6 \pm 12.8 and $14.3 \pm 2.5$, respectively) by flow cytometry analysis (Figure 2(a)). Furthermore, confocal microscopic analysis also supported the increased uptake of OVA when combined with the cationic liposomes (Figure 2(b)). These results revealed that cationic liposomes enhance the cellular uptake of OVA in murine dendritic cells, thereby acting as a carrier for antigen delivery.

\subsection{Mechanism of Enhanced Uptake of OVA by the Cationic Liposomes}

It is well-known that most endocytic pathways are energy-dependent and hence are inhibited at low temperatures (e.g. $4^{\circ} \mathrm{C}$ ) [28] [29]. Therefore, we have examined the temperature-dependency of OVA internalization in DC2.4 cells to understand the possible mechanisms of enhancement in uptake by the cationic liposomes. Figure 3 shows that OVA internalization is increased by the cationic liposomes at $4^{\circ} \mathrm{C}$ (MFI 26.7) which is otherwise inhibited (MFI 11.1), suggesting the involvement of energy-dependent endocytic pathways in the process. Various endocytic pathways, including phagocytosis, clathrin-mediated endocytosis (CME), lipid raft-mediated endocytosis, and macropinocytosis, are orchestrated to internalize a variety of molecules into the cell compartments [30] [31]. To identify the precise mechanism(s) of endocytosis mediating the increased uptake of OVA by the cationic liposomes, the effect of perturbation of endocytic pathways by various chemical inhibitors (Table 1) were examined (Figure 4). The 

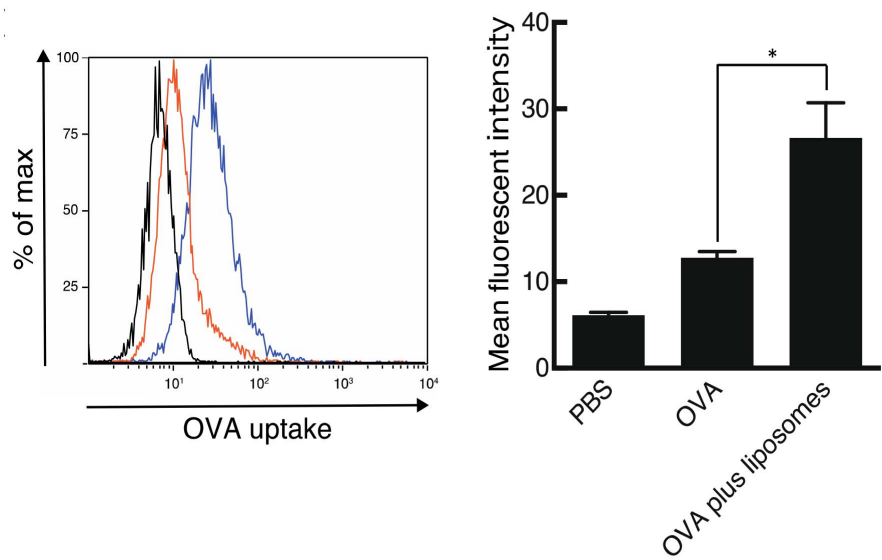

(a)

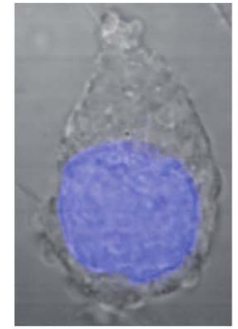

PBS

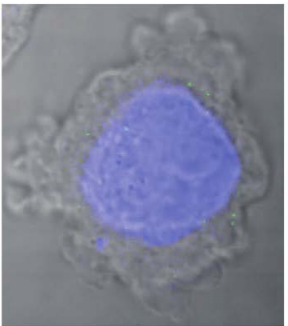

OVA

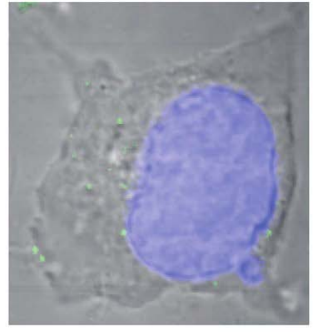

OVA plus liposomes

(b)

Figure 2. Antigen uptake by DC2.4 cells following co-culture with the cationic liposomes. (A) DC2.4 cells were treated with vehicle (black), FITC-OVA $(50 \mu \mathrm{g} / \mathrm{mL})$ (orange), or FITC-OVA $(50 \mu \mathrm{g} / \mathrm{mL})$ in combination with cationic liposomes $(20 \mathrm{nmol} / \mathrm{mL})$ (blue) for $1 \mathrm{~h}$. FITC-OVA uptake was then analyzed using flow cytometry as mean fluorescence intensities of the cells. The values are expressed as the mean \pm SEM of duplicate cultures from three independent experiments. Significance was evaluated by an unpaired $t$-test with Welch's correction: ${ }^{\star} p<0.05$, and (b). Images from confocal microscopy showing FITC-OVA uptake. FITC-OVA = green; DAPI = blue.

in vitro cellular toxicity of these inhibitors against DC2.4 cells were elucidated prior to perturbation experiments. The results indicated that these compounds did not affect the viability of DC2.4 cells at these concentrations used for the study (data not shown). Treatment with chlorpromazine and methyl- $\beta$-cyclodextrin significantly reduced the uptake of FITC-OVA into DC2.4 cells to $57.3 \%$ and $46.2 \%$, respectively, compared to that in the untreated group suggesting internalization of OVA by the DC2.4 cells via clathrin-mediated and lipid raft-mediated (cholesterol-dependent) endocytosis when co-incubated with the cationic liposomes. However, genistein, which inhibits caveolae-mediated (lipid raft-mediated) endocytic pathway, did not affect the uptake of OVA, implying that the endocytic pathway involved here might be clathrin-mediated and caveolae-independent lipid raft-mediated pathways, which is reported to be cholesterol-dependent previously [32] [33]. Further, we have studied the 

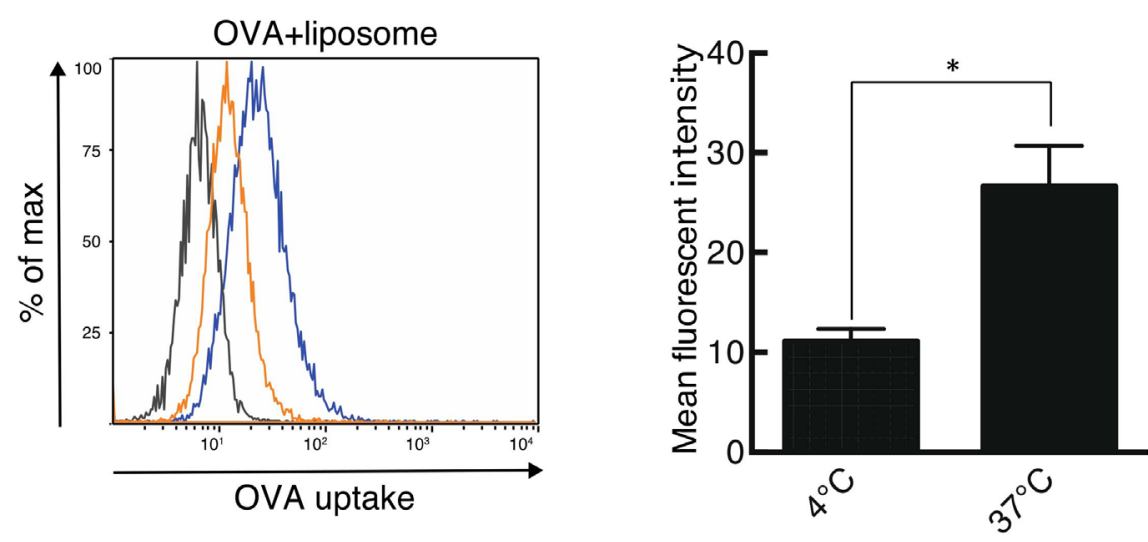

(a)

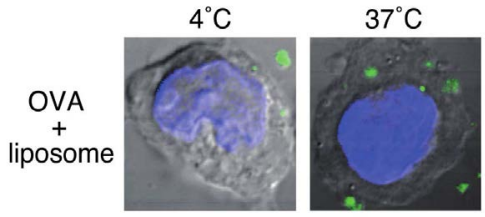

(b)

Figure 3. Effect of temperature on enhanced uptake of FITC-OVA by the cationic liposomes. DC2.4 cells were treated with vehicle (black) or FITC-OVA $(50 \mu \mathrm{g} / \mathrm{mL})$ in combination with cationic liposomes $\left(20 \mathrm{nmol} / \mathrm{mL}\right.$ ) for $1 \mathrm{~h}$ at $4^{\circ} \mathrm{C}$ (orange) or $37^{\circ} \mathrm{C}$ (blue). (a) FITC-OVA uptake was then analyzed using flow cytometry as mean fluorescence intensities of the cells. The values are expressed as the mean \pm SEM of duplicate cultures from three independent experiments. Significance was evaluated by an unpaired $t$-test with Welch's correction: ${ }^{\star} p<0.05$; (b) Confocal microscopy images for FITC-OVA uptake at different temperatures. FITC-OVA = green; DAPI = blue.

Table 1. Chemical inhibitors for endocytic pathways used in this study.

\begin{tabular}{cccc}
\hline Inhibitor & Pathway targeted & Final concentration & $\begin{array}{c}\text { Toxicity in } \\
\text { this condition }\end{array}$ \\
\hline Chlorpromazine & Clathrin-mediated endocytosis & $50 \mu \mathrm{M}$ & $(-)$ \\
Methyl- $\beta$-cyclodxtrin & Lipid raft & $31.25 \mu \mathrm{M}$ & $(-)$ \\
Amiloride & Macropinocytosis & $50 \mu \mathrm{M}$ & $(-)$ \\
Cytochalasin D & Phagocytosis & $2.5 \mu \mathrm{M}$ & $(-)$ \\
Genistein & Caveolin-mediated endocytosis & $25 \mathrm{nM}$ & $(-)$
\end{tabular}

endocytic pathways involved in the uptake of FITC-OVA and the cationic liposomes separately in the absence of each other. In the case of antigenic OVA, the uptake of protein was inhibited by the treatment of chlorpromazine (Figure 5), conforming the involvement of CME, as reported previously [34] [35], On the other hand, the pre-treatment of chlorpromazine, methyl- $\beta$-cyclodextrin, and cytochalasin D clearly suppressed cationic liposome uptake by DC2.4 cells (35.3\%, $24.1 \%$ and $23.3 \%$, respectively), indicating the implication of concerted mechanisms using clathrin-, cholesterol-, and actin-dependent pathways for the uptake of the cationic liposomes (Figure 6). Taken together, OVA uptake 


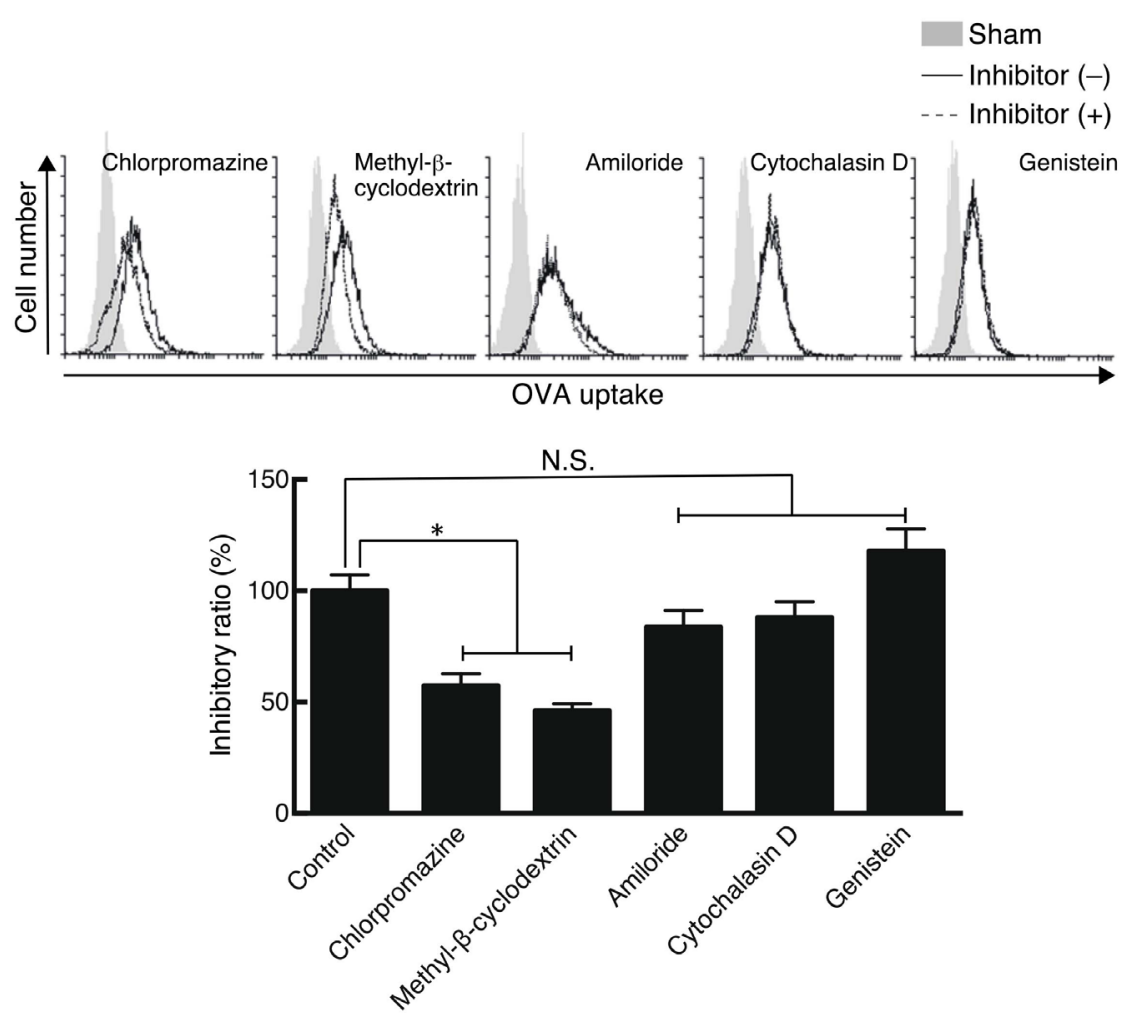

Figure 4. Effect of various chemical inhibitors of endocytic pathways on enhancement FITC-OVA uptake by the cationic liposomes. DC2.4 cells were pre-treated with various chemical inhibitors of endocytic pathways for $30 \mathrm{~min}$ at $37^{\circ} \mathrm{C}$, and then treated with vehicle (black), FITC-OVA $(50 \mu \mathrm{g} / \mathrm{mL})$ (orange), or FITC-OVA $(50 \mu \mathrm{g} / \mathrm{mL})$ in combination with cationic liposomes $\left(20 \mathrm{nmol} / \mathrm{mL}\right.$ ) (blue) for $1 \mathrm{~h}$ at $37^{\circ} \mathrm{C}$. FITC-OVA uptake was further analyzed using flow cytometry as mean fluorescence intensities of the cells. The values are expressed as the mean \pm SEM of duplicate cultures from three independent experiments. Significance was evaluated by an unpaired $t$-test with Welch's correction: ${ }^{\star} p<$ 0.05 , N.S.: not significant.

increased by the addition of the cationic liposomes may be mediated by clathrinand caveolae-independent lipid raft-mediated endocytic pathways.

\subsection{Complex Formation Is Crucial for the Uptake of OVA Enhanced by the Cationic Liposomes}

OVA is a typical acidic protein $(\mathrm{pI}=4.7)$ it and possesses a negative charge. Therefore, possible interaction of OVA with the cationic liposomes is via an electrostatic force and it is hypothesized that DC2.4 cells might engulf OVA as a complex with cationic liposomes. To verify this hypothesis, we have analyzed localization of OVA and the cationic liposomes when co-cultured with the cells. As shown in Figure 7, confocal imaging revealed that OVA (green) and liposomes (red) were largely co-localized in the cytosolic compartment of DC2.4 cells represented in yellow, implying that DC2.4 cells might uptake OVA as a complex with the cationic liposomes. To validate our hypothesis further, we compared OVA uptake in presence of cationic liposomes and after pre-treatment 


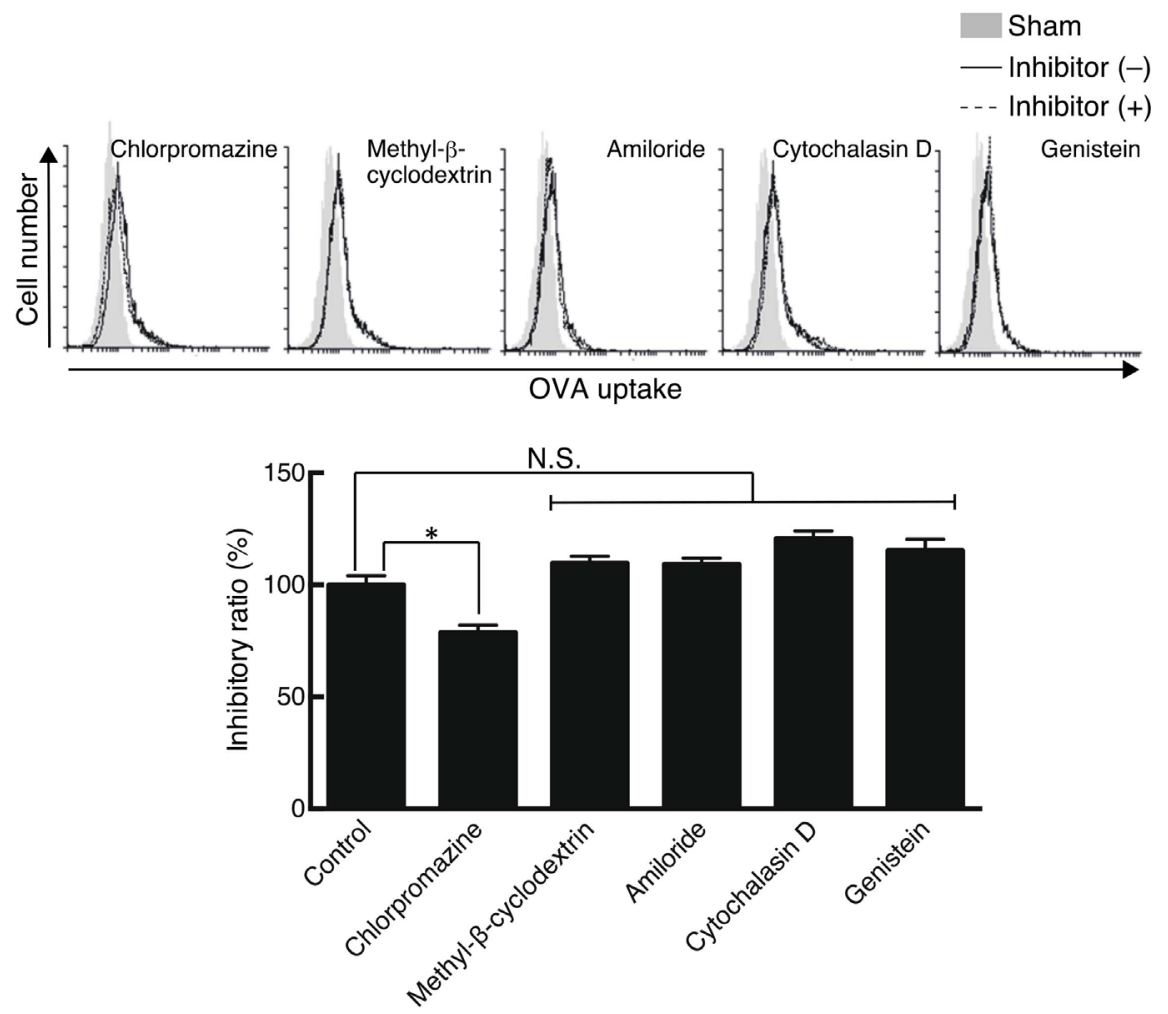

Figure 5. Mechanisms of uptake of FITC-OVA by DC2.4 cells. DC2.4 cells were pre-treated with various chemical inhibitors for endocytic pathways for $30 \mathrm{~min}$ at $37^{\circ} \mathrm{C}$, and then treated with vehicle or FITC-OVA $(50 \mu \mathrm{g} / \mathrm{mL})$ for $1 \mathrm{~h}$ at $37^{\circ} \mathrm{C}$. FITC-OVA uptake was further analyzed using flow cytometry as mean fluorescence intensities of the cells. The values are expressed as the mean \pm SEM of duplicate cultures from three independent experiments. Significance was evaluated by an unpaired $t$-test with Welch's correction: ${ }^{\star} p<0.05$, N.S.: not significant.

with cationic liposomes. In the latter case, the cells were treated with cationic liposomes first and OVA was added after washing out these liposomes. Figure 8 shows that increased uptake of OVA by liposomes could not be observed if DC2.4 cells are incubated separately with OVA and liposomes, indicating that the co-existence of OVA and liposomes is required for the enhancement in uptake of OVA mediated by the liposomes. Cumulatively, OVA interacted with the cationic liposomes maybe via electrostatic force and the uptake by DC2.4 cells is via both clathrin- and cholesterol-dependent endocytic pathways.

\section{Discussion}

We have reported that the cationic liposome composed of DOTAP and DC-chol acts as a potent adjuvant in mice. However, the underlying mechanism(s) were mostly unknown. In this study, we have investigated the pathways by which OVA is taken up by murine DCs cell line, DC2.4 cells, when co-cultured with the cationic liposomes, to evaluate whether specific endocytic pathways are contributing to antigenic delivery to APCs by the cationic liposomes. Our data herein show the following findings: 1) addition of cationic liposomes together 


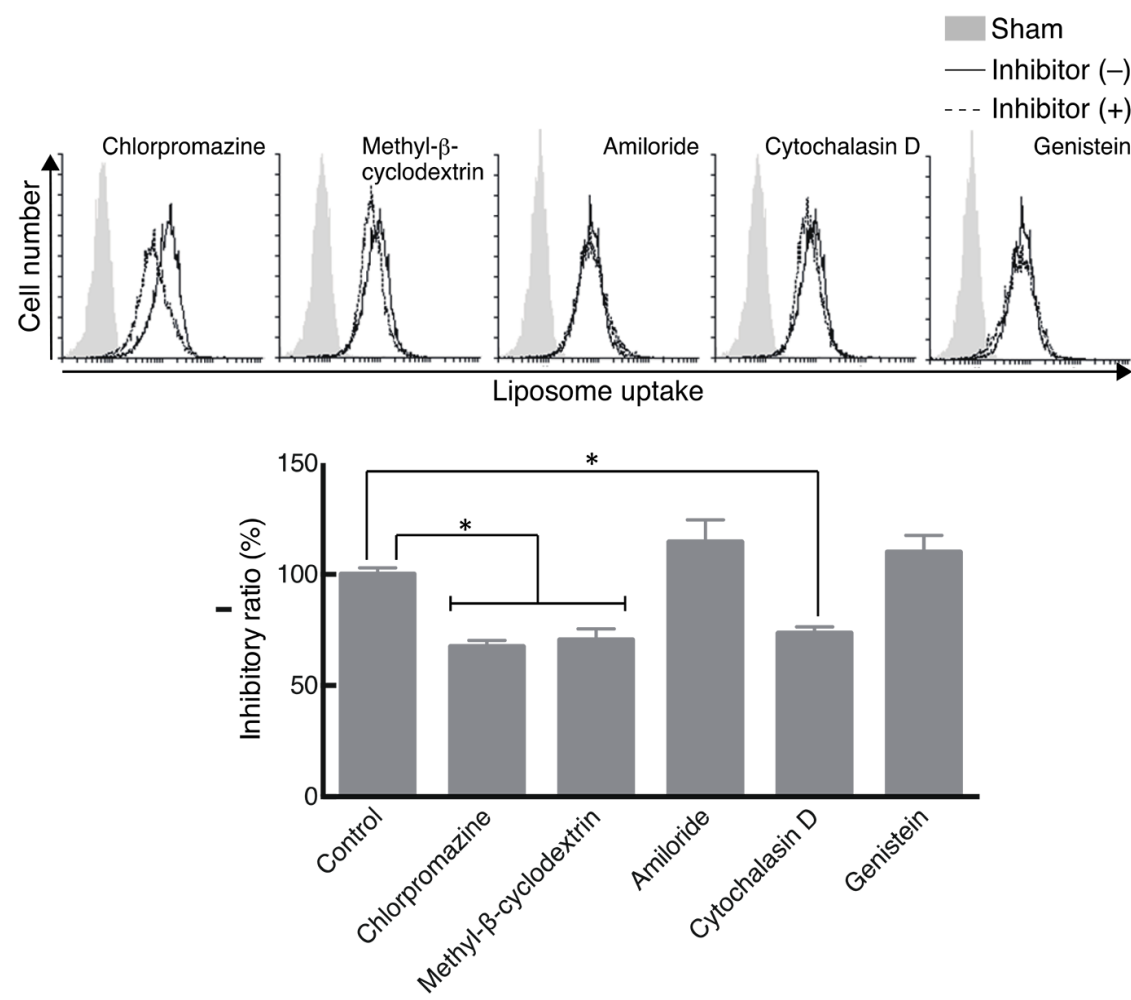

Figure 6. Mechanisms of uptake of cationic liposome by DC2.4 cells. DC2.4 cells were pre-treated with various chemical inhibitors for endocytic pathways for $30 \mathrm{~min}$ at $37^{\circ} \mathrm{C}$, and then treated with vehicle (black) or the cationic liposomes $(20 \mathrm{nmol} / \mathrm{mL})$ for $1 \mathrm{~h} \mathrm{at}$ $37^{\circ} \mathrm{C}$. The uptake of cationic liposome was further analyzed using flow cytometry as mean fluorescence intensities of the cells. The values are expressed as the mean \pm SEM of duplicate cultures from three independent experiments. Significance was evaluated by an unpaired $t$-test with Welch's correction: ${ }^{*} p<0.05$.
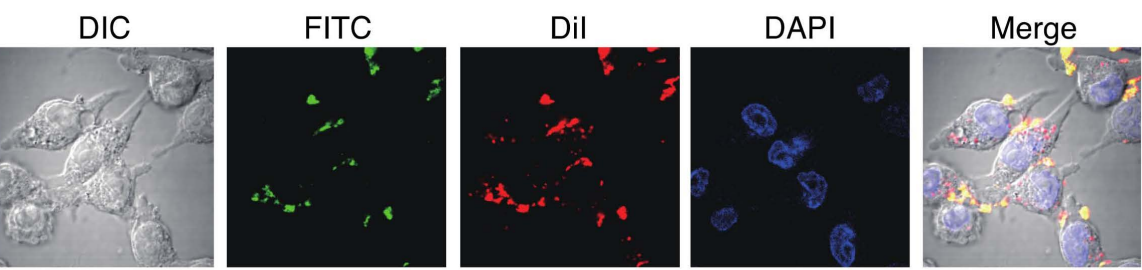

Figure 7. Co-localization of OVA and the cationic liposomes in DC2.4 cells. DC2.4 cells were treated with FITC-OVA $(50 \mu \mathrm{g} / \mathrm{mL})$ plusDiI-labeled cationic liposomes (20 $\mathrm{nmol} / \mathrm{mL}$ ) for $1 \mathrm{~h}$. Localization of FITC-OVA and DiI-liposome in DC2.4 cells were then analyzed using confocal microscopy. FITC-OVA = green; DiI-liposome = red; DAPI = blue; co-localization of FITC-OVA and DiI-liposome = yellow.

with OVA enhance the cellular uptake of antigen to murine dendritic cells, 2) increased uptake of OVA by the cationic liposomes is mediated by clathrin- and caveolae-independent lipid raft-mediated endocytic pathways, 3) DC2.4 cells internalized OVA and the cationic liposome when treated with them separately via clathrin-mediated and concerted mechanisms using clathrin-, caveolin-independent lipid raft-, and actin-dependent pathways, and 4) co-incubation of OVA and the cationic liposomes are essential for the enhanced uptake of 

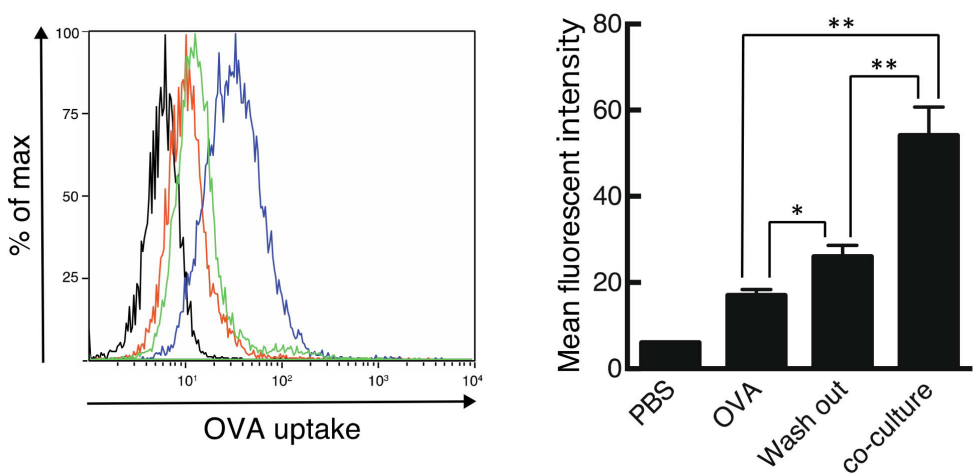

(a)
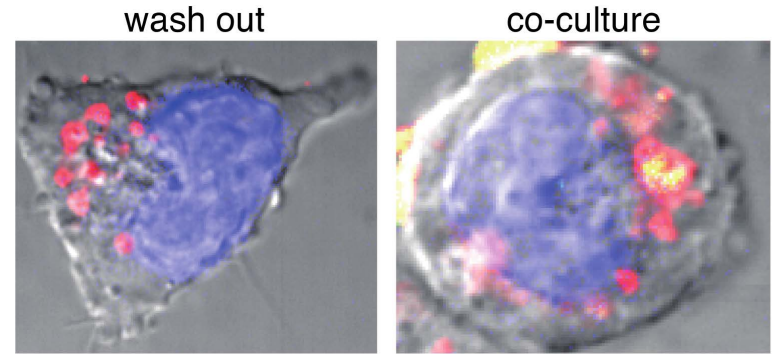

(b)

Figure 8. Coexistence of FITC-OVA and the cationic liposomes is crucial for enhanced uptake of FITC-OVA. (a) For co-culture experiment, DC2.4 cells were treated with vehicle (black), FITC-OVA $(50 \mu \mathrm{g} / \mathrm{mL})$ (orange), and FITC-OVA $(50 \mu \mathrm{g} / \mathrm{mL})$ in combination with cationic liposomes (20 $\mathrm{nmol} / \mathrm{mL}$ ) (blue) for $1 \mathrm{~h}$ at $37^{\circ} \mathrm{C}$. For wash-out experiment, DC2.4 cells were treated with cationic liposomes $(20 \mathrm{nmol} / \mathrm{mL})$ for $4 \mathrm{~h}$ at $37^{\circ} \mathrm{C}$. After washing the liposomes out, DC2.4 cells were incubated with FITC-OVA $(50 \mu \mathrm{g} / \mathrm{mL})$ for $1 \mathrm{~h}$ at $37^{\circ} \mathrm{C}$ (green). FITC-OVA uptake and the cationic liposome uptake was then analyzed using flow cytometry as mean fluorescence intensities of the cells. The values are expressed as the mean \pm SEM of duplicate cultures from three independent experiments. Significance was evaluated by an unpaired $t$-test with Welch's correction: ${ }^{\star} p<0.05$, (b) confocal microscopy images for wash-out and co-culture experiments. FITC-OVA = green; DiI-liposome = red; DAPI = blue; co-localization of FITC-OVA and DiI-liposome $=$ yellow .

OVA into DC2.4 cells. These results suggested that OVA interacted with the cationic liposomes presumably via electrostatic forces, was taken up by DC2.4 cells via both clathrin- and caveolae-independent but lipid raft-dependent endocytic pathways.

Recently there has been an increase in demand of safe and effective adjuvant which can enhance the immune response to the antigenic protein [36]. Generally, adjuvants exhibit their immune-enhancing activities against antigenic protein through the following mechanisms: 1) the depot effect that gradually releases antigen from adjuvant carriers at the site of administration, 2) activation of host innate immune responses, and 3) increased antigen uptake by APCs and presentation toward $\mathrm{T}$ cells. However, research suggested that the depot effect will be 
dispensable for adjuvant effects because the injection site can be immediately removed after immunization without any loss on the activities [37] [38]. While, several adjuvants, such as PEI, exert their effects via the release of damage-associated molecular patterns (DAMPs), resulting in activation of the innate immune system of the host by recognizing utilized in various pattern recognition receptors (PRRs) [39]. In the present study, we focused on whether the cationic liposomes, a potent adjuvant we have reported previously, act as an antigen delivery system into murine dendritic cells, DC2.4 cells. Numerous studies have reported that for example, a nanogel consisting of a cholesteryl-groupbearing pullulan induced antigen-specific antibody response by delivering antigens to DCs, implying adjuvant-free vaccination [12] [39]. These facts demonstrate that the antigen delivery to APCs is an important step for the induction of antigen-specific immune responses towards the antigen.

Since antigen internalization by APCs is essential for the effective antigen presentation onto MHC class II molecule, the delivery of antigens is a rational strategy for the initiation of adjuvant activities. We herein explored antigen delivery capacities of the cationic liposome to DC2.4 cells. We demonstrated that OVA in the presence of the cationic liposomes increased the cellular uptake to DC2.4 cells (Figure 2, Figure 7 and Figure 8). Perturbation studies using the chemical inhibitors for endocytic pathways indicated that the molecular mechanism(s) of enhanced uptake of OVA to DC2.4 cells rely on clathrin- and caveolae-independent lipid raft-mediated endocytic pathways (Figures 3-6). It is known that the interaction of nanoparticles like liposomes with cell membrane plays a significant role in the endocytic processes. These interactions depend upon hydrophobicity, size, and shape [40]. Recent evidences have shown that the cellular internalization of nanoparticles is highly influenced by the nature of their surface. For instance, cationic nanoparticles are readily taken up by cells compared to anionic nanoparticles [41]. Additionally, endocytic pathways involved in the process of internalization of nanoparticles by various cells are also influenced by nature of the surface of nanoparticles. Amphiphilic nanoparticles are preferentially taken up via lipid raft/caveolin-mediated process whereas, small polymeric particles are shown to enter the cell via clathrin- and caveolin-independent endocytosis [42] [43].

\section{Conclusions}

This study indicated that cellular uptake of antigens may be important for the adjuvant effects of the cationic liposome. Additionally, since the cationic liposomes exert cellular toxicity at higher concentrations both in vitro and in vivo, the use of these liposomes may pose a danger by releasing DAMPs at the site of administration. We are currently testing this possibility to understand the molecular mechanism(s) behind the adjuvant effects of cationic liposomes. This information may lead to development of efficient vaccine systems using liposomal formulations. 
In this study, we have shown that DOTAP/DC-chol liposomes enable dendritic cells to internalize antigenic proteins. This phenomenon might be mediated by clathrin- and caveolin-independent lipid raft-dependent endocytic pathways. Our results revealed, at least in part, that enhancement of antigen uptake by the cationic liposomes could be a possible mechanism behind the induction of antigen-specific immune responses by cationic these liposomes. Although further studies are needed to understand the precise mechanisms of adjuvant effects of DOTAP/DC-chol liposome, this approach is quite useful for the development of vaccine system to combat various diseases. One such study is in progress for the development of nasal pneumonia vaccine using liposomal formulations.

\section{Acknowledgements}

This study was supported in part by JSPS KAKENHI Grant Number 15K18935 (Grant-in-aid for Young Scientists (B) to R.T.); 16K08415 (Grant-in-Aid for Scientific Research C to Y.A. and R.T.).

\section{References}

[1] Fauci, A.S., Touchette, N.A. and Folkers, G.K. (2005) Emerging Infectious Diseases: a 10-Year Perspective from the National Institute of Allergy and Infectious Diseases. Emerging Infectious Diseases Journal, 11, 519-525. https://doi.org/10.3201/eid1104.041167

[2] Scully, I.L., Swanson, K., Green, L., Jansen, K.U. and Anderson, A.S. (2015) Anti-Infective Vaccination in the 21st Century-New Horizons for Personal and Public Health. Current Opinion in Microbiology, 27, 96-102. https://doi.org/10.1016/j.mib.2015.07.006

[3] Detmer, A. and Glenting, J. (2006) Live Bacterial Vaccines-A Review and Identification of Potential Hazards. Microbial Cell Factories, 5, 23. https://doi.org/10.1186/1475-2859-5-23

[4] Lauring, A.S., Jones, J.O. and Andino, R. (2010) Rationalizing the Development of Live Attenuated Virus Vaccines. Nature Biotechnology, 28, 573-579. https://doi.org/10.1038/nbt.1635

[5] Cho, H.W., Howard, C.R. and Lee, H.W. (2002) Review of an Inactivated Vaccine against Hantaviruses. Intervirology, 45, 328-333. https://doi.org/10.1159/000067925

[6] Foged, C. (2011) Subunit Vaccines of the Future: The Need for Safe, Customized and Optimized Particulate Delivery Systems. Therapeutic Delivery, 2, 1057-1077. https://doi.org/10.4155/tde.11.68

[7] Moyle, P.M. and Toth, I. (2013) Modern Subunit Vaccines: Development, Components, and Research Opportunities. ChemMedChem, 8, 360-376. https://doi.org/10.1002/cmdc.201200487

[8] Ada, G. (2001) Vaccines and Vaccination. New England Journal of Medicine, 345, 1042-1053. https://doi.org/10.1056/NEJMra011223

[9] Awate, S., Babiuk, L.A. and Mutwiri, G. (2013) Mechanisms of Action of Adjuvants. Frontiers in Immunology, 4, 114. https://doi.org/10.3389/fimmu.2013.00114

[10] Paulis, L.E., Mandal, S., Kreutz, M. and Figdor, C.G. (2013) Dendritic Cell-Based Nanovaccines for Cancer Immunotherapy. Current Opinion in Immunology, 25, 
389-395. https://doi.org/10.1016/j.coi.2013.03.001

[11] Reddy, S.T., Swartz, M.A. and Hubbell, J.A. (2006) Targeting Dendritic Cells with Biomaterials: Developing the Next Generation of Vaccines. Trends in Immunology, 27, 573-579. https://doi.org/10.1016/j.it.2006.10.005

[12] Kong, I.G., Sato, A., Yuki, Y., Nochi, T., Takahashi, H., Sawada, S., Mejima, M., Kurokawa, S., Okada, K., Sato, S., Briles, D.E., Kunisawa, J., Inoue, Y., Yamamoto, M., Akiyoshi, K. and Kiyono, H. (2013) Nanogel-Based PspA Intranasal Vaccine Prevents Invasive Disease and Nasal Colonization by Streptococcus pneumoniae. Infection and Immunity, 81, 1625-1634. https://doi.org/10.1128/IAI.00240-13

[13] Nochi, T., Yuki, Y., Takahashi, H., Sawada, S., Mejima, M., Kohda, T., Harada, N., Kong, I.G., Sato, A., Kataoka, N., Tokuhara, D., Kurokawa, S., Takahashi, Y., Tsukada, H., Kozaki, S., Akiyoshi, K. and Kiyono, H. (2010) Nanogel Antigenic Protein-Delivery System for Adjuvant-Free Intranasal Vaccines. Nature Materials, 9, 572-578. https://doi.org/10.1038/nmat2784

[14] Wegmann, F., Gartlan, K.H., Harandi, A.M., Brinckmann, S.A., Coccia, M., Hillson, W.R., Kok, W.L., Cole, S., Ho, L.P., Lambe, T., Puthia, M., Svanborg, C., Scherer, E.M., Krashias, G., Williams, A., Blattman, J.N., Greenberg, P.D., Flavell, R.A., Moghaddam, A.E., Sheppard, N.C. and Sattentau, Q.J. (2012) Polyethyleneimine Is a Potent Mucosal Adjuvant for Viral Glycoprotein Antigens. Nature Biotechnology, 30, 883-888. https://doi.org/10.1038/nbt.2344

[15] Endo-Takahashi, Y., Negishi, Y., Kato, Y., Suzuki, R., Maruyama, K. and Aramaki, Y. (2012) Efficient siRNA Delivery Using Novel siRNA-Loaded Bubble Liposomes and Ultrasound. International Journal of Pharmaceutics, 422, 504-509. https://doi.org/10.1016/j.ijpharm.2011.11.023

[16] Endo-Takahashi, Y., Negishi, Y., Nakamura, A., Suzuki, D., Ukai, S., Sugimoto, K., Moriyasu, F., Takagi, N., Suzuki, R., Maruyama, K. and Aramaki, Y. (2013) pDNA-Loaded Bubble Liposomes as Potential Ultrasound Imaging and Gene Delivery Agents. Biomaterials, 34, 2807-2813.

https://doi.org/10.1016/j.biomaterials.2012.12.018

[17] Motoyama, K., Nakashima, Y., Aramaki, Y., Hirayama, F., Uekama, K. and Arima, H. (2011) In Vitro Gene Delivery Mediated by Asialofetuin-Appended Cationic Liposomes Associated with gamma-Cyclodextrin into Hepatocytes. Journal of Drug Delivery, 2011, Article ID: 476137. https://doi.org/10.1155/2011/476137

[18] Negishi, Y., Endo-Takahashi, Y., Matsuki, Y., Kato, Y., Takagi, N., Suzuki, R., Maruyama, K. and Aramaki, Y. (2012) Systemic Delivery Systems of Angiogenic Gene by Novel Bubble Liposomes Containing Cationic Lipid and Ultrasound Exposure. Molecular Pharmaceutics, 9, 1834-1840. https://doi.org/10.1021/mp200554c

[19] Tada, R., Hidaka, A., Iwase, N., Takahashi, S., Yamakita, Y., Iwata, T., Muto, S., Sato, E., Takayama, N., Honjo, E., Kiyono, H., Kunisawa, J. and Aramaki, Y. (2015) Intranasal Immunization with DOTAP Cationic Liposomes Combined with DC-Cholesterol Induces Potent Antigen-Specific Mucosal and Systemic Immune Responses in Mice. PLoS ONE, 10, e0139785. https://doi.org/10.1371/journal.pone.0139785

[20] Tada, R., Muto, S., Iwata, T., Hidaka, A., Kiyono, H., Kunisawa, J. and Aramaki, Y. (2017) Attachment of class B CpG ODN onto DOTAP/DC-chol Liposome in Nasal Vaccine Formulations Augments Antigen-Specific Immune Responses in Mice. BMC Research Notes, 10, 68. https://doi.org/10.1186/s13104-017-2380-8

[21] Coester, C., Nayyar, P. and Samuel, J. (2006) In Vitro Uptake of Gelatin Nanoparticles by Murine Dendritic Cells and Their Intracellular Localisation. European 
Journal of Pharmaceuticsand Biopharmaceutics, 62, 306-314. https://doi.org/10.1016/j.ejpb.2005.09.009

[22] Harding, C.V. and Song, R. (1994) Phagocytic Processing of Exogenous Particulate Antigens by Macrophages for Presentation by Class I MHC Molecules. Journal of Immunology, 153, 4925-4933.

[23] Hermanson, G.T. (2008) Bioconjugate Techniques. Elsevier Academic Press, Amsterdam, Boston.

[24] Aramaki, Y., Takano, S., Arima, H. and Tsuchiya, S. (2000) Induction of Apoptosis in WEHI 231 Cells by Cationic Liposomes. Pharmaceutical Research, 17, 515-520. https://doi.org/10.1023/A:1007552529280

[25] Aramaki, Y., Takano, S. and Tsuchiya, S. (1999) Induction of Apoptosis in Macrophages by Cationic Liposomes. FEBS Letters, 460, 472-476. https://doi.org/10.1016/S0014-5793(99)01386-1

[26] Aramaki, Y., Takano, S. and Tsuchiya, S. (2001) Cationic Liposomes Induce Macrophage Apoptosis through Mitochondrial Pathway. Archives of Biochemistry and Biophysics, 392, 245-250. https://doi.org/10.1006/abbi.2001.2458

[27] Iwaoka, S., Nakamura, T., Takano, S., Tsuchiya, S. and Aramaki, Y. (2006) Cationic Liposomes Induce Apoptosis through p38 MAP Kinase-Caspase-8-Bid Pathway in Macrophage-like RAW264.7 Cells. Journal of Leukocyte Biology, 79, 184-191. https://doi.org/10.1189/jlb.0405181

[28] Steinman, R.M., Mellman, I.S., Muller, W.A. and Cohn, Z.A. (1983) Endocytosis and the Recycling of Plasma Membrane. Journal of Cell Biology, 96, 1-27.

https://doi.org/10.1083/jcb.96.1.1

[29] Pearse, B.M. and Bretscher, M.S. (1981) Membrane Recycling by Coated Vesicles. Annual Reviews of Biochemistry, 50, 85-101. https://doi.org/10.1146/annurev.bi.50.070181.000505

[30] Doherty, G.J. and McMahon, H.T. (2009) Mechanisms of Endocytosis. Annual Reviews of Biochemistry, 78, 857-902.

https://doi.org/10.1146/annurev.biochem.78.081307.110540

[31] Haucke, V. (2015) Cell Biology: On the Endocytosis Rollercoaster. Nature, 517, 446-447. https://doi.org/10.1038/nature14081

[32] Kirkham, M. and Parton, R.G. (2005) Clathrin-Independent Endocytosis: New Insights into Caveolae and Non-Caveolar Lipid Raft Carriers. Biochimica et BiophysicaActa, 1745, 273-286.

[33] Mayor, S. and Pagano, R.E. (2007) Pathways of Clathrin-Independent Endocytosis. Nature Reviews Molecular Cell Biology, 8, 603-612. https://doi.org/10.1038/nrm2216

[34] Autenrieth, S.E. and Autenrieth, I.B. (2009) Variable Antigen Uptake Due to Different Expression of the Macrophage Mannose Receptor by Dendritic Cells in Various Inbred Mouse Strains. Immunology, 127, 523-529. https://doi.org/10.1111/j.1365-2567.2008.02960.x

[35] Silva, J.M., Vandermeulen, G., Oliveira, V.G., Pinto, S.N., Rodrigues, C., Salgado, A., Afonso, C.A., Viana, A.S., Jerome, C., Silva, L.C., Graca, L., Preat, V. and Florindo, H.F. (2014) Development of Functionalized Nanoparticles for Vaccine Delivery to Dendritic Cells: A Mechanistic Approach. Nanomedicine, 9, 2639-2656. https://doi.org/10.2217/nnm.14.135

[36] Kunisawa, J., Gohda, M. and Kiyono, H. (2007) [Uniqueness of the Mucosal Immune System for the Development of Prospective Mucosal Vaccine.] Yakugaku- 
Zasshi, 127, 319-326. https://doi.org/10.1248/yakushi.127.319

[37] Hutchison, S., Benson, R.A., Gibson, V.B., Pollock, A.H., Garside, P. and Brewer, J.M. (2012) Antigen Depot Is Not Required for Alum Adjuvanticity. FASEB Journal, 26, 1272-1279. https://doi.org/10.1096/fj.11-184556

[38] Liang, F. and Lore, K. (2016) Local Innate Immune Responses in the Vaccine Adjuvant-Injected Muscle. Clinical and Translational Immunology, 5, e74. https://doi.org/10.1038/cti.2016.19

[39] Jounai, N., Kobiyama, K., Takeshita, F. and Ishii, K.J. (2012) Recognition of Damage-Associated Molecular Patterns Related to Nucleic Acids during Inflammation and Vaccination. Frontiers in Cellular and Infection Microbiology, 2, 168.

[40] Chakraborty, A. and Jana, N.R. (2015) Clathrin to Lipid Raft-Endocytosis via Controlled Surface Chemistry and Efficient Perinuclear Targeting of Nanoparticle. Journal of Physical Chemistry Letters, 6, 3688-3697. https://doi.org/10.1021/acs.jpclett.5b01739

[41] Hauck, T.S., Ghazani, A.A. and Chan, W.C. (2008) Assessing the Effect of Surface Chemistry on Gold Nanorod Uptake, Toxicity, and Gene Expression in Mammalian Cells. Small, 4, 153-159. https://doi.org/10.1002/smll.200700217

[42] Choi, C.H., Hao, L., Narayan, S.P., Auyeung, E. and Mirkin, C.A. (2013) Mechanism for the Endocytosis of Spherical Nucleic Acid Nanoparticle Conjugates. Proceedings of the National Academy of Sciences of the United States of America, 110, 7625-7630. https://doi.org/10.1073/pnas.1305804110

[43] Lai, S.K., Hida, K., Man, S.T., Chen, C., Machamer, C., Schroer, T.A. and Hanes, J. (2007) Privileged Delivery of Polymer Nanoparticles to the Perinuclear Region of Live Cells via a Non-Clathrin, Non-Degradative Pathway. Biomaterials, 28, 2876-2884. https://doi.org/10.1016/j.biomaterials.2007.02.021 